\title{
Interacción estrógenos-noradrenalina en la depresión
}

\author{
Nelly Maritza Vega-Rivera, ${ }^{1}$ Carolina López-Rubalcava, ${ }^{2}$ Nallely Paez-Martínez, ${ }^{1,3}$ \\ Mario Castro,' Erika Monserrat Estrada-Camarena'
}

\begin{abstract}
SUMMARY
Depression refers to a mood disorder characterized by deep sadness and loss of interest and pleasure. Epidemiologic studies show that this disorder represents a public health problem affecting $12 \%$ of the world population in a proportion of 2:1 of women to men. Depression is a complex disease in which it has been observed that the noradrenergic system appears to play an important role. Thus, a decrease in the noradrenergic tone, changes in noradrenaline (NA) synthesis, reduction in its turn-over, and modulation of its receptors can induce this disease. On the other hand, estrogens are a wide hormones family with multiple biologic functions which include those related to mood states. Clinical studies suggest that hormonal fluctuations, such as the premenstrual phase, puerperium and perimenopause, are associated with an increase in the vulnerability to depression. Conversely, estrogens have shown antidepressant effects in different preclinical models. Binding and electrophysiology studies suggest that estrogens are able to modulate noradrenergic transmission, through an increase of NA neurons' firing rate, a regulation of noradrenergic receptors and the synthesis and catabolism of this neurotransmitter. Additionally, behavioral studies support the interaction of estrogens with the noradrenergic system. Thus, the purpose of this review is to analyze the participation of noradrenalin, estrogens and their interaction in the treatment of depression in both, clinical and preclinical studies.
\end{abstract}

Key words: Estrogens, adrenaline, depression.

\section{Key words: Estrogens, adrenaline, depression.}

\begin{abstract}
RESUMEN
La depresión se define como un trastorno del estado de ánimo caracterizado por un estado de tristeza profunda y una pérdida de interés - placer. Este trastorno psiquiátrico afecta al $12 \%$ de la población mundial, siendo las mujeres quienes más la padecen. La depresión es una patología compleja, en la que se ha observado que el sistema noradrenérgico cumple un papel importante. Así, una disminución en el tono noradrenérgico, los cambios en la síntesis y el metabolismo de la noradrenalina (NA), así como en la modulación de sus receptores, pueden conducir a un estado depresivo. Por otro lado, los estrógenos son un grupo de hormonas gonadales con diversas funciones fisiológicas, incluidas las que se relacionan con los estados afectivos. Diversos estudios clínicos sugieren que las fluctuaciones hormonales, como la etapa premenstrual, el puerperio y la perimenopausia, se asocian con un aumento en la vulnerabilidad a presentar depresión y se ha demostrado que los estrógenos presentan efectos antidepresivos en diversos modelos conductuales. En estudios electrofisiológicos y de unión de ligando se reporta que los estrógenos son capaces de modular la transmisión noradrenérgica a través de diferentes mecanismos, los cuales incluyen un aumento en la frecuencia de disparo de las neuronas noradrenérgicas, la regulación de la densidad de los receptores noradrenérgicos, así como en los procesos de síntesis y metabolismo de este neurotransmisor. Además, diversos estudios conductuales han aportado información que apoya la participación de los estrógenos en la modulación del sistema noradrenérgico e incluso se ha propuesto que a través de esta vía podrían inducir sus efectos antidepresivos. De esta forma, el propósito de esta revisión es analizar, a nivel clínico y preclínico, la participación de la noradrenalina y de los estrógenos, y la relación entre ambos en el tratamiento de la depresión.
\end{abstract}

Palabras clave: Estrógenos, adrenalina, depresión.

\section{LA DEPRESIÓN}

La depresión es un estado afectivo de tristeza que se presenta como respuesta a una variedad de factores biológicos, genéticos y psicosociales. ${ }^{1}$ Según el Manual Diagnóstico y Estadís- tico de Trastornos Mentales, ${ }^{2}$ la depresión se define como un trastorno del estado de ánimo caracterizado por un estado de tristeza profunda y una pérdida de interés o placer. Estos síntomas, que perduran durante al menos dos semanas y están presentes la mayor parte del día, suelen estar acompañados

\footnotetext{
Laboratorio de Neuropsicofarmacología. Dirección de Neurociencias. Instituto Nacional de Psiquiatría Ramón de la Fuente Muñiz.

Departamento de Farmacobiología, CINVESTAV.

Sección de Posgrado e Investigación. Escuela Superior de Medicina, IPN.
}

Correspondencia: Dra. Erika Estrada-Camarena. Laboratorio de Neuropsicofarmacología, Dirección de Neurociencias. Instituto Nacional de Psiquiatría Ramón de la Fuente Muñiz. Calz. México-Xochimilco 101, San Lorenzo Huipulco, Tlalpan, 14370, México, DF. Tel: (52-55) 4160-5053. Fax: (52-55) 5655-9980.

E-mail: estrada@imp.edu.mx

Recibido: 8 de agosto de 2012. Aceptado: 19 de abril de 2013. 
por al menos cuatro de los síntomas siguientes: a) cognitivos, como pérdida de interés, dificultad para concentrarse, baja autoestima, culpa, ideas suicidas; b) conductuales, como retraso o agitación psicomotora, retraimiento, tendencia al llanto y c) somáticos, como trastornos del sueño (insomnio o hipersomnia), aumento o disminución del apetito, pérdida o aumento de peso, fatiga y disminución de la libido. ${ }^{1}$

Los estudios epidemiológicos muestran que la depresión es un problema de salud pública que afecta a un $12 \%$ de la población mundial, siendo las mujeres quienes más la padecen, en una relación de 2:1 respecto de los hombres., De acuerdo con la Organización Mundial de la Salud, la depresión ocupa el cuarto sitio entre las diez causas de morbilidad a nivel mundial, y se estima que para el año 2020 será la segunda causa de incapacidad. ${ }^{5}$

\section{LA NORADRENALINA Y LA DEPRESIÓN}

\subsection{La noradrenalina}

La noradrenalina (NA) es el neurotransmisor de la mayor parte de las fibras simpáticas posganglionares y de muchas neuronas centrales. Esta catecolamina deriva del aminoácido tirosina y tiene como intermediarios de su síntesis a la L-3,4dihidroxifenilalanina (DOPA) y a la dopamina. Las enzimas catalíticas responsables de este proceso son la tirosina hidroxilasa, la dopa-descarboxilasa y la dopamina $\beta$-hidroxilasa. La NA es almacenada en las terminales nerviosas dentro de vesículas, y liberada en la hendidura sináptica por exocitosis. Algunas de las moléculas liberadas se difunden para unirse a receptores pos-sinápticos y activarlos, induciendo de esta manera una respuesta fisiológica. Otras moléculas de NA pueden ocupar autorreceptores, así como recapturarse por el transportador en neuronas presinápticas para ser realmacenadas, o bien, ser metabolizadas por la monoaminooxidasa $\mathrm{A}$ (MAO-A) o por la catecol-O-metil-transferasa (COMT) ${ }^{6}$

Los cuerpos celulares y las fibras que contienen NA se originan en el locus coeruleus y otras áreas noradrenérgicas inferiores, como el núcleo del tracto solitario o los núcleos retículo-laterales. Desde estos núcleos surgen dos grandes vías de proyección ascendente: la vía noradrenérgica dorsal y la vía noradrenérgica ventral. Ambas vías controlan el nivel de actividad córtico-subcortical, principalmente por su proyección a la corteza, al sistema límbico (hipocampo, amígdala y septum) y al diencéfalo (tálamo e hipotálamo). ${ }^{7}$ De esta forma, a través de estas proyecciones ascendentes, se piensa que la NA está relacionada con el control de las emociones y del estado de ánimo. ${ }^{1}$

\subsection{Los receptores noradrenérgicos}

Los receptores noradrenérgicos se dividen en dos familias: los a y los $\beta .{ }^{8}$ La familia de receptores $\alpha_{1}$ se subdivide en $\alpha_{1 \mathrm{~A}^{\prime}}$ $\alpha_{1 \mathrm{~B}}$ y $\alpha_{1 \mathrm{D}^{\prime}}$ mientras que los $\alpha_{2}$ se subdividen en $\alpha_{2 \mathrm{~A}^{\prime}} \alpha_{2 \mathrm{~B}^{\prime}} \alpha_{2 \mathrm{C}} \mathrm{y}$ $\alpha_{2 \mathrm{D}}$. En cuanto a la familia de los receptores $\beta$ se conocen tres subtipos: $\beta_{1}, \beta_{2}$ y $\beta_{3}$. Los receptores $\alpha_{1}$ y los $\beta$ se localizan en la postsinápsis, en tanto que los receptores $\alpha_{2}$ se encuentran localizados tanto en la presinápsis como en la postsinápsis. ${ }^{1,6}$

Cuando se estimulan las neuronas noradrenérgicas, la NA se libera hacia la hendidura sináptica e interactúa con sus receptores postsinápticos $\alpha_{1} \mathrm{o} \beta$. Cuando las concentraciones de NA en el espacio sináptico son altas, una porción de la NA liberada interactúa con los receptores presinápti$\cos$ (autorreceptores) $\alpha_{2}$, produciendo un proceso de retroalimentación negativa o autoinhibición, que sirve para reducir la transmisión noradrenérgica. ${ }^{6}$

Las tres familias de receptores adrenérgicos se acoplan a proteínas $\mathrm{G}$. Los receptores $\alpha_{1}$ son generalmente excitadores, acoplados a la fosfolipasa C (PLC), de tal manera que al ser estimulados inducen la formación de inositol trifosfato (IP3) y $\mathrm{Ca}^{2+}$. Por otro lado, los receptores $\alpha_{2}$ disminuyen la formación de AMPc, ya que están acoplados negativamente a la adenilatociclasa. A su vez, los receptores $\beta$ se encuentran acoplados positivamente a la adenilatociclasa de tal manera que su estimulación incrementa la formación de AMPc. ${ }^{6,9}$

\subsection{El papel del sistema noradrenérgico en la depresión}

Aunque el tratamiento de la depresión se ha enfocado en gran parte en el neurotransmisor serotonina (5-HT), se sabe que el sistema noradrenérgico también se encuentra involucrado, tanto en la patogénesis como en el tratamiento de esta enfermedad. ${ }^{10-12}$ En cuanto a la etiología, se ha observado que los pacientes deprimidos muestran una reducción en la actividad de NA, al verse reducidos los niveles del metabolito de este neurotransmisor, el3-metoxi-4-hidroxifenilglicol (MHPG). ${ }^{13,14}$ Además, los pacientes tratados con fármacos inhibidores selectivos de NA muestran una recaída en los efectos depresivos al ser administrados con un inhibidor de la síntesis de NA. ${ }^{14}$ Por otro lado, en estudios post-mortem de suicidas se ha encontrado que el locus coeruleus presenta un marcado incremento tanto en los niveles de la enzima tirosina hidroxilasa, como en la densidad de los autorreceptores $\alpha_{2}$ adrenérgicos, ${ }^{15}$ lo que sugiere un agotamiento de la NA en ciertas regiones del cerebro. ${ }^{16}$ Además, algunos estudios muestran la existencia de un polimorfismo del receptor adrenérgico $\alpha_{2 C^{\prime}}$ el cual se ha asociado a las alteraciones en la actividad neuronal, en la amígdala y el cíngulo, en respuesta a información emocional (presentación de rostros tristes) en pacientes deprimidos. ${ }^{17}$

En términos del tratamiento, se ha observado que la administración de inhibidores selectivos de la recaptura de noradrenalina, como la reboxetina, es tan eficaz en el tratamiento de la depresión como los inhibidores de la recaptura de serotonina, como la fluoxetina. ${ }^{18,19}$ Incluso se ha observado que la reboxetina ha tenido buenos resultados en pacientes deprimidos que no responden al tratamiento con fluoxeti- 
na. ${ }^{20}$ De igual forma, la duloxetina y la venlafaxina - que son inhibidores duales, ya que bloquean la recaptura tanto de noradrenalina como de serotonina - han demostrado ser eficaces en el tratamiento de la depresión mayor. ${ }^{21-24}$ Además, en la búsqueda de nuevas estrategias para el tratamiento de la depresión, se ha sugerido que la coadministración de antagonistas de los autorreceptores $\alpha_{2}$ adrenérgicos con inhibidores de la recaptura de noradrenalina aumenta la concentración de noradrenalina en varias áreas cerebrales e induce efectos antidepresivos. ${ }^{25}$ Este aumento se ha observado tanto con inhibidores selectivos de noradrenalina, como la desipramina, la reboxetina y la atomoxetina, así como con el uso de inhibidores no selectivos de la recaptura de noradrenalina, como la sibutramina, la duloxetina y la venlafaxina. De hecho, se ha propuesto que este aumento de la NA pudiera reducir el tiempo en que se observa el efecto antidepresivo. ${ }^{25}$

\section{LOS ESTRÓGENOS Y LA DEPRESIÓN}

\subsection{Los estrógenos}

El término estrógenos se le da al grupo de hormonas gonadales con diversas funciones fisiológicas en varios tejidos y tipos celulares. Aunque al principio se consideró que se trataba de las hormonas responsables de la función del sistema reproductor femenino, hoy en día se sabe que los estrógenos también están implicados en funciones cerebrales relacionadas con estados afectivos. ${ }^{26,27}$

A los estrógenos los podemos clasificar en tres diferentes grupos:

1. Estrógenos Naturales: Son compuestos esteroides de 18 átomos de carbono, e incluyen la estrona $\left(\mathrm{E}_{1}\right)$, el $17 \beta$ estradiol $\left(\mathrm{E}_{2}\right)$ y el estriol $\left(\mathrm{E}_{3}\right)$. Los ovarios son la principal fuente de $\mathrm{E}_{2}$ en mujeres premenopáusicas, mientras que la mayoría de la $\mathrm{E}_{1}$ y del $\mathrm{E}_{3}$ se forma en el hígado, a partir de $E_{2^{\prime}}$ o en tejidos periféricos a partir de androstendiona. En mujeres postmenopáusicas, los principales precursores de la producción de estrógenos en los tejidos periféricos son la androstenediona, la testosterona y la $\mathrm{E}_{1} \cdot{ }^{27}$

2. Estrógenos conjugados o semisintéticos: Estos compuestos son producto de la alteración química de los estrógenos naturales. Los estrógenos sintéticos incluyen la premarina (estrógeno conjugado de equino compuesto de sulfato de $\mathrm{E}_{1} \mathrm{y}$ de los estrógenos equilina y equilenina), el etinilestradiol $\left(\mathrm{EE}_{2}\right)$ (levonorgenestrel), el dietilestilbestrol (DES), el clorotrianiseno, el dienestrol, el fosfestrol, el mestranol, el fosfato de poliestradiol y el quinestrol. ${ }^{27}$

3. Fitoestrógenos: Son compuestos polifenólicos no esteroideos, que son extraídos de plantas y que poseen actividad estrogénica. Los fitoestrógenos se encuentran en una variedad de plantas y alimentos como la soya (genisteína y daidzeína), el trigo, el trébol rojo (Promensil) y los cacahuates. Basados en su estructura química, los podemos clasificar en flavonoides, estilbenoides y lignanos. ${ }^{27,28}$

\subsection{Los receptores a estrógenos}

El efecto de los estrógenos está mediado por dos receptores intracelulares específicos (ER $\alpha$ y ER $\beta$ ) y un receptor membranal, denominado GPR30. La activación de un gen para los receptores ER $\alpha$ y ER $\beta$ puede dar lugar a múltiples proteínas del mismo receptor. Los mecanismos implicados en esta diversidad incluyen los cambios epigenéticos y la metilación de genes que codifican para estos receptores, el splicing alternativo del RNA que produce múltiples isoformas del RNAm de cada receptor, y los múltiples sitios para la iniciación o traducción del RNAm de estos receptores. En general, la activación de los receptores ER $\alpha$ y ER $\beta$ conduce a la activación transcripcional de genes blanco. Por otro lado, el receptor GPR30 es un receptor de siete dominios transmembranales acoplado a proteínas G y cuya activación resulta en una movilización de $\mathrm{Ca}^{2+}$ intracelular y en la síntesis de IP3. ${ }^{27}$

\subsection{La participación de los estrógenos en la depresión}

Diversas líneas de investigación señalan que los estrógenos cumplen un papel importante en la modulación de la depresión. ${ }^{29-31}$ La evidencia clínica sugiere que las fluctuaciones hormonales drásticas que se presentan a lo largo de la vida, como la etapa premenstrual, el puerperio y la perimenopausia, se asocian a una mayor vulnerabilidad en la aparición de alteraciones psiquiátricas, como la depresión. ${ }^{32,33}$ Se ha especulado incluso que estas alteraciones psiquiátricas están asociadas a los cambios en los niveles de esteroides sexuales, como los estrógenos, los cuales ejercen una modulación importante en las funciones cerebrales que producen una modificación en el estado de ánimo y la conducta. ${ }^{31,33}$ En este sentido, se ha observado que los episodios de depresión durante la menopausia se acompañan con la caída de los niveles de estradiol. De igual forma, se ha observado que la eliminación de la terapia de reemplazo hormonal, en mujeres postmenopáusicas, parece estar asociada con la presentación de cuadros depresivos. ${ }^{31,34}$ Además, se ha demostrado que el estradiol es de utilidad en el tratamiento de los síntomas de la depresión postparto. ${ }^{35}$

En línea con lo reportado en la clínica, los estudios preclínicos han encontrado que los estrógenos, como el E2 y el EE2, administrados por vía subcutánea o en estructuras cerebrales específicas (como el hipocampo), inducen efectos antidepresivos en varios modelos animales. ${ }^{26,36-39}$ Además se ha observado que este efecto es de larga duración, ya que se mantiene de 48 a $72 \mathrm{~h} .{ }^{40} \mathrm{El}$ efecto de estos compuestos se ha relacionado directamente con los receptores a estrógenos, ya que la administración de antagonistas específicos de estos receptores, como el RU 58668 y el ICI 182-780, bloquea su efecto antidepresivo. ${ }^{41,42}$

Por otra parte, estudios con ratones knockout del receptor $E R \beta^{43} y$ con moduladores selectivos de estos receptores, 
como el coumestrol o el diarilpropionitrilo, apoyan la participación de los receptores ER $\beta$ en la regulación de las conductas depresivas. ${ }^{44}$

\section{LA INTERACCIÓN ESTRÓGENOS- SISTEMA NORADRENÉRGICO EN LA DEPRESIÓN}

Diferentes investigaciones, incluidos estudios de unión de ligando ${ }^{45,46}$ y registros electrofisiológicos, ${ }^{47,48}$ han mostrado que los estrógenos pueden modular la neurotrasmisión noradrenérgica a nivel del Sistema Nervioso Central. La modulación de este sistema se lleva a cabo a través de la liberación de NA, la regulación de sus receptores, así como en los procesos de síntesis y de eliminación del neurotransmisor.

En cuanto a la liberación de NA y la regulación de los receptores, se ha observado que el $\mathrm{E}_{2}$ administrado a ratas hembras ovariectomizadas aumenta la frecuencia de disparo de las neuronas noradrenérgicas que se proyectan hacia el área preóptica y el área hipotalámica anterior. ${ }^{48}$ Asímismo, el $\mathrm{E}_{2}$, el DES y el mestranol redujeron la densidad de receptores $\alpha$-adrenérgicos en estructuras como el hipotálamo, la corteza frontal y el núcleo del tracto solitario. ${ }^{45,46,49-51}$ De igual forma, el $E_{2}$ aumenta la actividad de los receptores $\alpha_{1}$ en neuronas del área preóptica, además de que disminuye la expresión del RNAm para el receptor $\alpha_{2}{ }^{47}$ Finalmente, también se ha reportado que el tratamiento crónico con $\mathrm{E}_{2}$ reduce la respuesta de los receptores $\beta$-adrenérgicos. ${ }^{52}$

Por otro lado, en relación con la recaptura, la síntesis y el metabolismo de NA, estudios in vitro han mostrado que $\mathrm{E}_{2}, \mathrm{EE}_{2}$, DES y algunos catecol-estrógenos, como el 2-hidroxi$\mathrm{EE}_{2}\left(2-\mathrm{OH}-\mathrm{EE}_{2}\right)$ y el 2-hidroxi-E 1 (2-OHE), inhiben los sitios de recaptura de NA en sinaptosomas de la corteza cerebral y del hipotálamo de la rata, ${ }^{53}$ lo que tiene como consecuencia un aumento en los niveles de NA en el espacio sináptico. En línea con estos hallazgos, estudios realizados por Hiemke et al. ${ }^{54}$ muestran que una administración aguda de $\mathrm{E}_{2}$ a ratas ovariectomizadas disminuye la tasa de recaptura de NA en el hipotálamo e incrementa los niveles del RNAm de la tirosina hidroxilasa en el locus coeruleus, aumentando así los niveles de NA. ${ }^{55}$ Finalmente, se ha reportado que los estrógenos también aumentan los niveles de NA por inhibición de la actividad de la MAO. .6,57 $^{2}$

De forma interesante, algunos estudios preclínicos (en el modelo de depresión de nado forzado) apoyan la idea de que los estrógenos inducen efectos antidepresivos a través de la modulación de la trasmisión noradrenérgica. Por ejemplo, se ha observado que el $\mathrm{EE}_{2}$ presenta un perfil conductual similar a los antidepresivos mixtos de la recaptura de noradrenalina y de serotonina, como la duloxetina y la venlafaxina. ${ }^{40,58,59}$ De igual forma, se ha observado que el estrógeno $\mathrm{EE}_{2}$ facilita el efecto antidepresivo de un inhibidor selectivo de la recaptura de NA, la desipramina. ${ }^{60}$ Estos re- sultados han permitido proponer que el efecto tipo antidepresivo de $\mathrm{EE}_{2}$ se relaciona con una activación del sistema noradrenérgico, además del serotonérgico.

En la misma línea de investigación se ha sugerido que la interacción del $\mathrm{EE}_{2}$ con el sistema noradrenérgico podría ser mediada por receptores $\alpha_{2}$-adrenérgicos, ya que el idazoxan, un antagonista selectivo de estos receptores, es capaz de bloquear el efecto tipo antidepresivo inducido por $\mathrm{EE}_{2}{ }^{4}{ }^{4}$ Aunado a estos estudios, recientemente se encontró que el DSP4, una neurotoxina que destruye selectivamente las terminales nerviosas noradrenérgicas originadas en el locus coeruleus ${ }^{61}$ fue capaz de cancelar el efecto tipo antidepresivo inducido por el $\mathrm{EE}_{2}$ en la prueba de nado forzado. ${ }^{42}$

En conjunto, estos hallazgos sugieren que los estrógenos pueden facilitar la transmisión noradrenérgica al: 1 . inducir el aumento en la síntesis de NA; 2 . reducir la recaptura de NA, mejorando así la disponiblidad de NA o 3. mediante un mecanismo que involucra los dos anteriores. Es importante considerar que, de acuerdo con la información precedente, resulta evidente que el efecto antidepresivo de los estrógenos se asocia con una mezcla entre su efecto sobre el sistema noradrenérgico y su acción directa sobre los receptores a estrógenos.

En conclusión, la depresión es una patología compleja que involucra sistemas de neurotransmisión como el noradrenérgico. Diversas investigaciones han confirmado la participación de los estrógenos en la modulación de la depresión, y esta investigación apunta a que esta regulación se realiza a través de la neurotransmisión noradrenérgica. Este conjunto de información sitúa a los estrógenos como nuevos candidatos para ampliar nuestro conocimiento sobre la fisiopatología de la depresión, así como en el descubrimiento de nuevas estrategias terapéuticas en el tratamiento de esta patología.

\section{REFERENCIAS}

1. Stahl SM. Essential psychopharmaology; neuroscientific basis and practical applications. Cambridge: Cambridge University Press; 2000.

2. Diagnostic and Statistical Manual of Mental Disorders DSM-IV-TR. Cuarta edición. Washington: American Psychiatric Association; 2000.

3. Andrade L, Caraveo-Anduaga JJ, Berglund P, Bijl RV et al. The epidemiology of major depressive episodes: results from the International Consortium of Psychiatric Epidemiology (ICPE) Surveys. Int J Methods Psychiatr Res 2003;12(1):3-21.

4. Caraveo-Anduaga JJ, Colmenares-Bermudez E, Saldivar-Hernandez GJ. [Gender differences in alcohol consumption in Mexico City]. Salud Publica Mex 1999;41(3):177-188.

5. Gender and women's mental health. En: World Health Organization; 2008.

6. Feldman RS, Meyer JS, Quenzer LF. Principles of neuropsychopharmacology. Massachussetts: Sinauer Associates; 1997.

7. Foote SL, Bloom FE, Aston-Jones G. Nucleus locus ceruleus: new evidence of anatomical and physiological specificity. Physiol Rev 1983;63(3):844-914.

8. Bylund DB, Eikenberg DC, Hieble JP, Langer SZ et al. International union of pharmacology nomenclature of adrenoceptors. Pharmacol $\operatorname{Rev} 1994 ; 46(2): 121-136$. 
9. Jewell-Motz EA, Liggett SB. G protein-coupled receptor kinase specificity for phosphorylation and desensitization of alpha2-adrenergic receptor subtypes. J Biol Chem 1996;271(30):18082-18087.

10. Blier $P$, Galzin AM, Langer SZ. Interaction between serotonin uptake inhibitors and alpha-2 adrenergic heteroreceptors in the rat hypothalamus. J Pharmacol Exp Ther 1990;254(1):236-244.

11. Delgado PL, Price LH, Miller HL, Salomon RM et al. Rapid serotonin depletion as a provocative challenge test for patients with major depression: relevance to antidepressant action and the neurobiology of depression. Psychopharmacol Bull 1991;27(3):321-330.

12. Delgado PL, Miller HL, Salomon RM, Licinio J et al. Monoamines and the mechanism of antidepressant action: effects of catecholamine depletion on mood of patients treated with antidepressants. Psychopharmacol Bull 1993;29(3):389-396.

13. Meana JJ, Barturen F, Garcia-Sevilla JA. Alpha 2-adrenoceptors in the brain of suicide victims: increased receptor density associated with major depression. Biol Psychiatry 1992;31(5):471-490.

14. Miller HL, Delgado PL, Salomon RM, Berman R et al. Clinical and biochemical effects of catecholamine depletion on antidepressant-induced remission of depression. Arch Gen Psychiatry 1996;53(2):117-128.

15. Ordway GA. Pathophysiology of the locus coeruleus in suicide. Ann N Y Acad Sci 1997;836:233-252.

16. Glavin GB. Stress and brain noradrenaline: a review. Neurosci Biobehav Rev 1985;9(2):233-243.

17. Neumeister A, Drevets WC, Belfer I, Luckenbaugh DA et al. Effects of a alpha 2C-adrenoreceptor gene polymorphism on neural responses to facial expressions in depression. Neuropsychopharmacology 2006;31(8):1750-1756.

18. Andreoli V, Caillard V, Deo RS, Rybakowski JK et al. Reboxetine, a new noradrenaline selective antidepressant, is at least as effective as fluoxetine in the treatment of depression. J Clin Psychopharmacol 2002;22(4):393-399.

19. Messer $T$, Schmauss $M$, Lambert-Baumann J. Efficacy and tolerability of reboxetine in depressive patients treated in routine clinical practice. CNS Drugs 2005;19(1):43-54.

20. Fava M, McGrath PJ, Sheu WP. Switching to reboxetine: an efficacy and safety study in patients with major depressive disorder unresponsive to fluoxetine. J Clin Psychopharmacol 2003;23(4):365-369.

21. Cowen PJ, Ogilvie AD, Gama J. Efficacy, safety and tolerability of duloxetine $60 \mathrm{mg}$ once daily in major depression. Curr Med Res Opin 2005;21(3):345-356.

22. Frampton JE, Plosker GL. Duloxetine: a review of its use in the treatment of major depressive disorder. CNS Drugs 2007;21(7):581-609.

23. Rabasseda X. Duloxetine: a new serotonin/noradrenaline reuptake inhibitor for the treatment of depression. Drugs Today (Barc) 2004;40(9):773-790.

24. Wellington $\mathrm{K}$, Perry $\mathrm{CM}$. Venlafaxine extended-release: a review of its use in the management of major depression. CNS Drugs 2001;15(8):643-669.

25. Invernizzi RW, Garattini S. Role of presynaptic alpha2-adrenoceptors in antidepressant action: recent findings from microdialysis studies. Prog Neuropsychopharmacol Biol Psychiatry 2004;28(5):819-827.

26. Rachman IM, Unnerstall JR, Pfaff DW, Cohen RS. Estrogen alters behavior and forebrain c-fos expression in ovariectomized rats subjected to the forced swim test. Proc Natl Acad Sci U S A 1998;95(23):13941-13946.

27. Smiley DA, Khalil RA. Estrogenic compounds, estrogen receptors and vascular cell signaling in the aging blood vessels. Curr Med Chem 2009;16(15):1863-1887.

28. Lethaby A, Hogervorst E, Richards M, Yesufu A et al. Hormone replacement therapy for cognitive function in postmenopausal women. Cochrane Database Syst Rev 2008(1):CD003122.

29. Panay N, Studd JW. The psychotherapeutic effects of estrogens. Gynecol Endocrinol 1998;12(5):353-365.

30. Soares CN, Frey BN. Is there a role for estrogen in treating depression during menopause? J Psychiatry Neurosci 2010;35(6):E6-7.

31. Schmidt PJ, Rubinow DR. Sex hormones and mood in the perimenopause. Ann N Y Acad Sci 2009;1179:70-85.
32. Parry BL, Newton RP. Chronobiological basis of female-specific mood disorders. Neuropsychopharmacology 2001;25(5 Supl):S102-S108.

33. Soares CN, Poitras JR, Prouty J. Effect of reproductive hormones and selective estrogen receptor modulators on mood during menopause. Drugs Aging 2003;20(2):85-100.

34. Stewart DE, Rolfe DE, Robertson E. Depression, estrogen, and the Women's Health Initiative. Psychosomatics 2004;45(5):445-447.

35. Soares CN, Almeida OP, Joffe H, Cohen LS. Efficacy of estradiol for the treatment of depressive disorders in perimenopausal women: a double-blind, randomized, placebo-controlled trial. Arch Gen Psychiatry 2001;58(6):529-534.

36. Bernardi M, Vergoni AV, Sandrini M, Tagliavini S et al. Influence of ovariectomy, estradiol and progesterone on the behavior of mice in an experimental model of depression. Physiol Behav 1989;45(5):10671068.

37. Galea LA, Wide JK, Barr AM. Estradiol alleviates depressive-like symptoms in a novel animal model of post-partum depression. Behav Brain Res 2001;122(1):1-9.

38. Okada M, Hayashi N, Kometani M, Nakao K et al. Influences of ovariectomy and continuous replacement of 17beta-estradiol on the tail skin temperature and behavior in the forced swimming test in rats. Jpn J Pharmacol 1997;73(1):93-96.

39. Dhir A, Kulkarni SK. Antidepressant-like effect of 17beta-estradiol: involvement of dopaminergic, serotonergic, and (or) sigma-1 receptor systems. Can J Physiol Pharmacol 2008;86(10):726-735.

40. Estrada-Camarena E, Fernandez-Guasti A, López-Rubalcava C. Antidepressant-like effect of different estrogenic compounds in the forced swimming test. Neuropsychopharmacology 2003;28(5):830-838.

41. Estrada-Camarena E, López-Rubalcava C, Fernández-Guasti A. Facilitating antidepressant-like actions of estrogens are mediated by 5 HT1A and estrogen receptors in the rat forced swimming test. Psychoneuroendocrinology 2006;31(8):905-914.

42. López-Rubalcava C, Vega Rivera N, Cruz-Martínez JJ, Estrada-Camarena E. En: 12th Biennial meeting of the European Behavioral Pharmacology Society 2007; Tübingen, Alemania; 2007.

43. Walf AA, Frye CA. A review and update of mechanisms of estrogen in the hippocampus and amygdala for anxiety and depression behavior. Neuropsychopharmacology 2006;31(6):1097-1111.

44. Walf AA, Frye CA. Administration of estrogen receptor beta-specific selective estrogen receptor modulators to the hippocampus decrease anxiety and depressive behavior of ovariectomized rats. Pharmacol Biochem Behav 2007;86(2):407-414.

45. Wagner HR, Davies JN. Decreased beta-adrenergic responses in the female rat brain are eliminated by ovariectomy: correlation of [3H]dihydroalprenolol binding and catecholamine stimulated cyclic AMP levels. Brain Res 1980;201(1):235-239.

46. Wilkinson M, Herdon HJ. Diethylstilbestrol regulates the number of alpha- and beta-adrenergic binding sites in incubated hypothalamus and amygdala. Brain Res 1982;248(1):79-85.

47. Wagner EJ, Ronnekleiv OK, Kelly MJ. The noradrenergic inhibition of an apamin-sensitive, small-conductance $\mathrm{Ca} 2+$-activated $\mathrm{K}+$ channel in hypothalamic gamma-aminobutyric acid neurons: pharmacology, estrogen sensitivity, and relevance to the control of the reproductive axis. J Pharmacol Exp Ther 2001;299(1):21-30.

48. Kaba H, Saito H, Otsuka K, Seto K et al. Effects of estrogen on the excitability of neurons projecting from the noradrenergic A1 region to the preoptic and anterior hypothalamic area. Brain Res 1983;274(1):156159.

49. Yoshioka M, Matsumoto M, Numazawa R, Togashi $H$ et al. Changes in the regulation of 5-hydroxytryptamine release by alpha2-adrenoceptors in the rat hippocampus after long-term desipramine treatment. Eur J Pharmacol 1995;294(2-3):565-370.

50. Karkanias GB, Li CS, Etgen AM. Estradiol reduction of alpha 2-adrenoceptor binding in female rat cortex is correlated with decreases in alpha 2A/D-adrenoceptor messenger RNA. Neuroscience 1997;81(3):593-597. 
51. Shackelford DP Jr, McConnaughey MM, Iams SG. The effects of estradiol and mestranol on alpha-adrenoceptors in select regions of the rat brain. Brain Res Bull 1988;21(2):329-333.

52. Carlberg KA, Fregly MJ. Catecholamine excretion and beta-adrenergic responsiveness in estrogen-treated rats. Pharmacology 1986;32(3):147-156.

53. Ghraf R, Michel M, Hiemke C, Knuppen R. Competition by monophenolic estrogens and catecholestrogens for high-affinity uptake of [3H](-)-norepinephrine into synaptosomes from rat cerebral cortex and hypothalamus. Brain Res 1983;277(1):163-168.

54. Hiemke C, Bruder D, Poetz B, Ghraf R. Sex-specific effects of estradiol on hypothalamic noradrenaline turnover in gonadectomized rats. Exp Brain Res 1985;59(1):68-72.

55. Serova L, Rivkin M, Nakashima A, Sabban EL. Estradiol stimulates gene expression of norepinephrine biosynthetic enzymes in rat locus coeruleus. Neuroendocrinology 2002;75(3):193-200.

56. Holschneider DP, Kumazawa T, Chen K, Shih JC. Tissue-specific effects of estrogen on monoamine oxidase A and B in the rat. Life Sci 1998;63(3):155-160.
57. Ortega-Corona BG, Valencia-Sanchez A, Kubli-Garfias C, Anton-Tay F et al. Hypothalamic monoamine oxidase activity in ovariectomized rats after sexual behavior restoration. Arch Med Res 1994;25(3):337-340.

58. Reneric JP, Lucki I. Antidepressant behavioral effects by dual inhibition of monoamine reuptake in the rat forced swimming test. Psychopharmacology (Berl) 1998;136(2):190-197.

59. Connor TJ, Kelliher P, Shen Y, Harkin A et al. Effect of subchronic antidepressant treatments on behavioral, neurochemical, and endocrine changes in the forced-swim test. Pharmacol Biochem Behav 2000;65(4):591-597.

60. Estrada-Camarena E, Fernández-Guasti A, López-Rubalcava C. Interaction between estrogens and antidepressants in the forced swimming test in rats. Psychopharmacology (Berl) 2004;173(1-2):139-145.

61. Jonsson G, Hallman H, Ponzio F, Ross S. DSP4 (N-(2-chloroethyl)-Nethyl-2-bromobenzylamine)--a useful denervation tool for central and peripheral noradrenaline neurons. Eur J Pharmacol 1981;72(2-3):173-188.

Artículo sin conflicto de intereses 